\title{
Tracheostomy in trauma patients with rib fractures
}

\author{
Alexander Fokin ${ }^{1} \cdot$ Joanna Wycech $^{1,2} \cdot$ Kyle Chin Shue $^{3} \cdot$ Ryan Stalder $^{5} \cdot$ Jose Lozada $^{2} \cdot$ Ivan Puente $^{1,2,3,4}$
}

Received: 8 November 2018 / Accepted: 13 May 2019 / Published online: 22 May 2019

(c) Springer-Verlag GmbH Germany, part of Springer Nature 2019

\begin{abstract}
Purpose Patients with rib fractures (RF) may require prolonged mechanical ventilation and tracheostomy. Indications for tracheostomy in trauma patients with RF remain debatable. The goal was to delineate characteristics of patients who underwent tracheostomy due to thoracic versus extra-thoracic causes, such as maxillofacial-mandibular injury (MFM), traumatic brain injury (TBI), and cervical vertebrae trauma (CVT), and to analyze clinical outcomes. The predictive values of chest trauma scoring systems for tracheostomy were also evaluated. We hypothesized that tracheostomized patients were more severely injured with more ribs fractured and had more pulmonary co-injuries.

Methods Retrospective review included 471 patients with RF admitted to two Level 1 trauma centers. Patients with tracheostomy $(n=124,26.3 \%)$ were compared to patients with endotracheal intubation $(n=347,73.7 \%)$. Analyzed variables included age, gender, injury severity score (ISS), Glasgow Coma Scale, number of ribs fractured, total fractures of ribs, prevalence of bilateral rib fractures, flail chest, clavicle fractures, MFM, TBI, CVT, co-injuries, comorbidities, RF treatment options, hospital length of stay (HLOS), intensive care unit LOS (ICULOS), duration of mechanical ventilation (DMV).

Results Tracheostomized compared to intubated patients had statistically higher ISS, more ribs fractured, total fractures of the ribs, bilateral and clavicle fractures, MFM, spine, chest, and orthopedic co-injuries and longer HLOS, ICULOS and DMV. Tracheostomy for thoracic reasons was performed in 64 patients (51.6\%) and for extra-thoracic reasons in 60 patients (48.4\%). Mean tracheostomy timing was 9.9 days and was significantly shorter in the extra-thoracic compared to the thoracic group $(8.0$ versus 11.6 days, $p<0.001)$. All chest trauma scoring system values were significantly higher in tracheostomized patients. Predictive values of scoring systems for tracheostomy increased in patients with thoracic trauma only.

Conclusions A quarter of mechanically ventilated patients with RF required tracheostomy. Tracheostomized compared to intubated patients were more severely injured with more ribs fractured and were intubated longer. An increased amount of $\mathrm{RF}$ was associated with an increase in tracheostomies, especially for thoracic reasons.
\end{abstract}

Keywords Tracheostomy $\cdot$ Rib fractures $\cdot$ Chest trauma $\cdot$ Rib fracture scoring systems

Alexander Fokin

Alexander.Fokin@tenethealth.com

1 Division of Trauma and Critical Care Services, Delray Medical Center, 5352 Linton Boulevard, Delray Beach, FL 33484, USA

2 Division of Trauma and Critical Care Services, Broward Health Medical Center, 1600 S Andrews Ave, Fort Lauderdale, FL 33316, USA

3 Charles E. Schmidt College of Medicine, Department of Surgery, Florida Atlantic University, 777 Glades Rd, Boca Raton, FL 33431, USA

4 Herbert Wertheim College of Medicine, Department of Surgery, Florida International University, 11200 SW 8th St, Miami, FL 33199, USA

5 Wake Forest University, 1834 Wake Forest Rd, Winston-Salem, NC 27109, USA

\section{Introduction}

Rib fractures (RF) are considered a marker of severe thoracic injuries [1-4]. Even minor thoracic trauma may cause functional impact with $22.8 \%$ of patients having severe or moderate disability at 90 days after trauma [5]. The number of rib fractures threshold for unfavorable outcomes varies depending on patients cohorts, but overall multiple rib fractures are associated with increased morbidity and mortality $[6,7]$. It was reported that the number of fractured ribs that best predicted the appearance of pleuropulmonary complications was three or more [8]. Treatment of patients with chest trauma and rib fractures may necessitate mechanical ventilation. Being a part of airway management for patients in need of prolonged mechanical ventilation, tracheostomy 
is a subject of interest for practicing surgeons. However, the information regarding tracheostomy in trauma patients with rib fractures is divisive and scattered among different subgroups of trauma patients [9-11].

The goal of our study was to delineate characteristics of trauma patients with rib fractures who underwent tracheostomy and to correlate these data with the number of fractured ribs and clinical outcomes. The focus was to distinguish between tracheostomies performed due to thoracic versus extra-thoracic causes, such as traumatic brain injury (TBI), maxillofacial-mandibular injury (MFM) and cervical vertebrae trauma (CVT). The second objective was to assess the predictive value for tracheostomy of different chest trauma scoring systems: rib fracture score (RFS), chest wall trauma score (CWTS), chest trauma score (CTS) and RibScore (RS). We also aimed to provide practical recommendations for trauma surgeons concerning the administration of tracheostomy in chest trauma patients with multiple rib fractures. We hypothesized that tracheostomized patients were more severely injured with more ribs fractured and had more pulmonary co-injuries.

\section{Methods}

This IRB approved retrospective chart review study included 471 patients with radiologically confirmed rib fractures (computed tomography scans and X-rays) admitted to two Level 1 trauma centers from January 2012 to December 2017 who required mechanical ventilation. Patients who underwent tracheostomy ( $n=124,26.3 \%)$ were compared to patients with endotracheal intubation $(n=347,73.7 \%)$.

Data were extracted from the trauma registries and patient electronic medical records. International Classification of Diseases 9th and 10th Revisions were used to identify rib fractures and other abstracted variables. Co-injuries were also identified by AIS cutoff value of $\geq 2$. Analyzed variables included age, gender, injury severity score (ISS), Glasgow Coma Scale (GCS), number of ribs fractured, number of total fractures of the ribs, prevalence of displaced rib fractures, bilateral rib fractures, presence of flail chest, $1 \mathrm{st}$ rib fractures, sternal fractures, clavicle fractures, MFM, TBI, chest, spine, pulmonary, orthopedic and abdominal co-injuries, comorbidities, incidence of epidural analgesia, surgical stabilization of rib fractures (SSRF), hospital length of stay (HLOS), intensive care unit length of stay (ICULOS), duration of mechanical ventilation (DMV), and tracheostomy timing (time from intubation to tracheostomy).

The values of RFS, CWTS, CTS and RS were calculated as previously described [12-16]. Variables for all four scoring systems (although in different combinations and different values assigned for each variable) included age, number of ribs fractured, number of total fractures of the ribs, bilateral rib fractures, displaced rib fractures, 1 st rib fractures, presence of flail chest, and pulmonary contusion [12-16].

IBM SPSS software version 25.0 (IBM, Armonk, NY) was used for statistical analyses, which included group characteristics, logistic regression and receiver operator characteristics (ROC). For group characteristics, calculations included categorical variables that were analyzed with $\chi^{2}$ test. The variable means were compared using twosided independent samples $t$ test for normally distributed variables, and the Mann-Whitney U test for non-normally distributed variables. One-way ANOVA with Tukey's post hoc test was used to compare mean ISS values between the groups of patients with different number of ribs fractured. Statistical significance was assumed when calculated $p$ value was below 0.05 . The ROC optimal cut point analysis based on the point closest to $(0-1)$ corner methodology was used for prediction of recommendations for tracheostomy. Binary logistic regression analysis was further used to account for confounders (significantly different independent variables) to determine the prediction power of tracheostomy.

The ROC area under the curve (AUC) analysis was used to determine prediction values $(c)$ of rib scoring systems for need for tracheostomy. The following scale was used to describe predictive power: $0.5<c<0.6$ denoted failed prediction, $0.6<c<0.7$ poor prediction, $0.7<c<0.8$ fair prediction, $0.8<c<0.9$ good prediction, and $0.9<c<1$ an excellent prediction.

The primary outcome was the distinctive features of chest trauma patients that prompted tracheostomy in patients with multiple rib fractures. The secondary outcomes were practical recommendations for trauma surgeons regarding indications for tracheostomy and the assessment of different chest trauma scoring systems in the prediction of tracheostomy.

\section{Results}

Among 2792 patients with rib fractures admitted to two Level 1 Trauma Centers, 471 (16.9\%) subjects were endotracheally intubated and 124 of those (26.3\% from intubated or $4.4 \%$ from all patients with RF) underwent tracheostomy.

The study design and patient selection flowchart is presented in Fig. 1.

Motor vehicle accidents were the leading mechanism of injury $(85.5 \%$ in the tracheostomized group and $76.7 \%$ in the intubated group), followed by falls ( $12.1 \%$ in tracheostomized and $13.5 \%$ in intubated patients). All patients in the study had blunt chest trauma.

Comparison between intubated and tracheostomized patients with RF is presented in Table 1. Tracheostomized patients had statistically significantly higher ISS, more ribs fractured, higher total number of fractures of the ribs, more bilateral RF and clavicle fractures. MFM co-injuries as well 
Fig. 1 Study design and patient selection flowchart

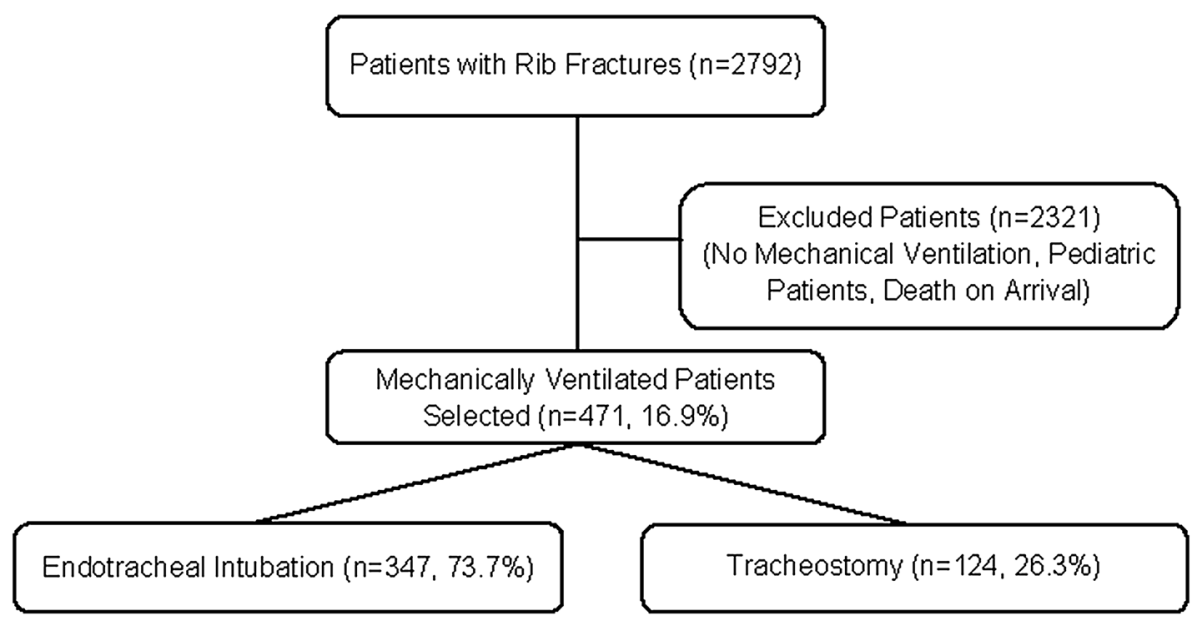

Table 1 Characteristics of intubated and tracheostomized patients with rib fractures

\begin{tabular}{|c|c|c|c|}
\hline & Intubation $(n=347)$ & Tracheostomy $(n=124)$ & $p$ values \\
\hline Age (yrs) & 49.7 & 50.3 & 0.7 \\
\hline Male gender $(\%)$ & $36.0 \%$ & $45.2 \%$ & 0.07 \\
\hline Injury severity score (ISS) & 23.1 & 27.5 & $<0.001^{*}$ \\
\hline Number of ribs fractured (Avg.) & 4.0 & 5.2 & $0.003^{*}$ \\
\hline Number of total rib fractures (Avg.) & 4.6 & 5.9 & $0.006^{*}$ \\
\hline Patients with displaced rib fractures & $22.8 \%$ & $30.6 \%$ & 0.08 \\
\hline Bilateral rib FX & $20.7 \%$ & $29.5 \%$ & $0.04 *$ \\
\hline Flail Chest & $4.6 \%$ & $8.1 \%$ & 0.1 \\
\hline 1st rib FX & $27.8 \%$ & $28.2 \%$ & 0.9 \\
\hline Glasgow come scale (ED) & 9.6 & 9.4 & 0.7 \\
\hline TBI (intracranial hemorrhage) co-injuries & $40.1 \%$ & $46.0 \%$ & 0.3 \\
\hline Maxillofacial co-injuries & $22.5 \%$ & $40.3 \%$ & $<0.001^{*}$ \\
\hline Spine co-injuries & $47.0 \%$ & $64.5 \%$ & $0.001 *$ \\
\hline Chest co-injuries (includes clavicle, sternal, scapula, diaphragm FXs) & $30.0 \%$ & $44.4 \%$ & $0.004 *$ \\
\hline Clavicle fracture & $15.3 \%$ & $26.6 \%$ & $0.005^{*}$ \\
\hline Sternal fracture & $5.5 \%$ & $9.7 \%$ & 0.1 \\
\hline Pulmonary co-injuries (includes hemo-/pneumothorax, pulmonary contusion) & $78.7 \%$ & $85.5 \%$ & 0.1 \\
\hline Pulmonary contusion & $49.3 \%$ & $57.3 \%$ & 0.1 \\
\hline Pneumonia & $16.4 \%$ & $35.5 \%$ & $<0.001^{*}$ \\
\hline Cardiovascular co-injuries & $13.3 \%$ & $16.1 \%$ & 0.4 \\
\hline Abdominal co-injuries & $33.1 \%$ & $37.1 \%$ & 0.4 \\
\hline Orthopedic co-injuries & $55.3 \%$ & $66.1 \%$ & $0.04 *$ \\
\hline Timing of tracheostomy (from intubation to tracheostomy) & N/A & 9.9 & N/A \\
\hline DMV (mean days) (intubation + tracheostomy) & 6.0 & 26.8 & $<0.001^{*}$ \\
\hline ICULOS (mean days) & $8.2(n=345)$ & $20.8(n=123)$ & $<0.001^{*}$ \\
\hline HLOS (mean days) & 15.1 & 39.3 & $<0.001 *$ \\
\hline
\end{tabular}

$F X$ fractures, $T B I$ traumatic brain injury, $D M V$ duration of mechanical ventilation, ICULOS intensive care unit length of stay, $H L O S$ hospital length of stay

*Denotes a significant difference

as spine, chest, and orthopedic trauma were significantly more common in tracheostomized patients. HLOS, ICULOS and DMV were significantly longer in tracheostomized patients. Mean tracheostomy timing was 9.9 days. 
Isolated rib fractures were present in $8.1 \%(n=28)$ of intubated patients and in $4.8 \%(n=6)$ of tracheostomized patients with rib fractures.

The top five comorbidities in the intubated group were: (1) hypertension (22.8\%), (2) smoking (12.7\%), (3) diabetes $(10.7 \%)$, (4) psychiatric disorder (7.8\%), and (5) alcoholism (6.6\%). In tracheostomized patients, the top five comorbidities were: (1) hypertension (25\%), (2) diabetes $(11.3 \%)$, (3) smoking $(7.3 \%)$, (4) drug dependence $(5.6 \%)$, and (5) alcoholism (4.8\%). Comorbidities were almost identical in both groups, and there was no statistically significant difference in the distribution of different types of comorbidities $(p=0.5)$.

Stratification of intubated and tracheostomized patients by the number of fractured ribs is presented in Fig. 2. It shows incremental increase in the ratio of tracheostomized versus intubated patients from $1: 4$ to $1: 3$ to $1: 2$ as the number of rib fractures increases.

For further analysis, all tracheostomized patients $(n=124)$ were divided into two groups. The extra-thoracic group included 60 patients $(48.4 \%)$ and the thoracic group included remaining 64 of the tracheostomized patients (51.6\%). Comparison between these two groups is presented in Table 2. The thoracic group of tracheostomized patients had significantly more fractured ribs, total number of rib fractures, sternal fractures, and higher prevalence of pulmonary contusion, chest co-injuries, pulmonary and orthopedic co-injuries. Timing of tracheostomy was statistically significantly shorter in the extra-thoracic compared to the thoracic group (8.0 days versus 11.6 days, $p<0.001$ ).

All intubated patients $(n=347)$ were also stratified into thoracic and extra-thoracic groups, and the thoracic group of intubated patients $(n=214)$ was compared to the thoracic group of tracheostomized patients $(n=64)$. Results of the comparison are presented in Table 3 . The thoracic group of tracheostomized patients compared to the thoracic group of intubated patients had higher ISS, number of fractured ribs, number of total rib fractures, displaced rib fractures, bilateral rib fractures, clavicle fractures, sternal fractures, chest, spine, pulmonary and orthopedic co-injuries, higher incidence of pulmonary contusion and pneumonia. HLOS, ICULOS and DMV were longer in tracheostomized patients.

Only 2 out of the 124 tracheostomized patients (1.6\%) and 6 out of the 347 intubated patients $(1.7 \%)$ received epidural analgesia.

Surgical stabilization of rib fractures (SSRF) was performed in 5 tracheostomized patients (4\%) with an average of 7.4 fractured ribs and in 14 intubated patients $(4 \%)$ with an average of 7.0 fractured ribs. All patients with SSRF underwent tracheostomy for thoracic reasons.

Clavicle plating was performed in 4 tracheostomized patients with ipsilateral rib fractures, with all subjects having the 1 st rib fractured. Clavicle plating was also performed in 11 intubated patients with ipsilateral rib fractures including 5 subjects with the 1 st rib fractured. None of the patients with plated clavicle fractures had SSRF.

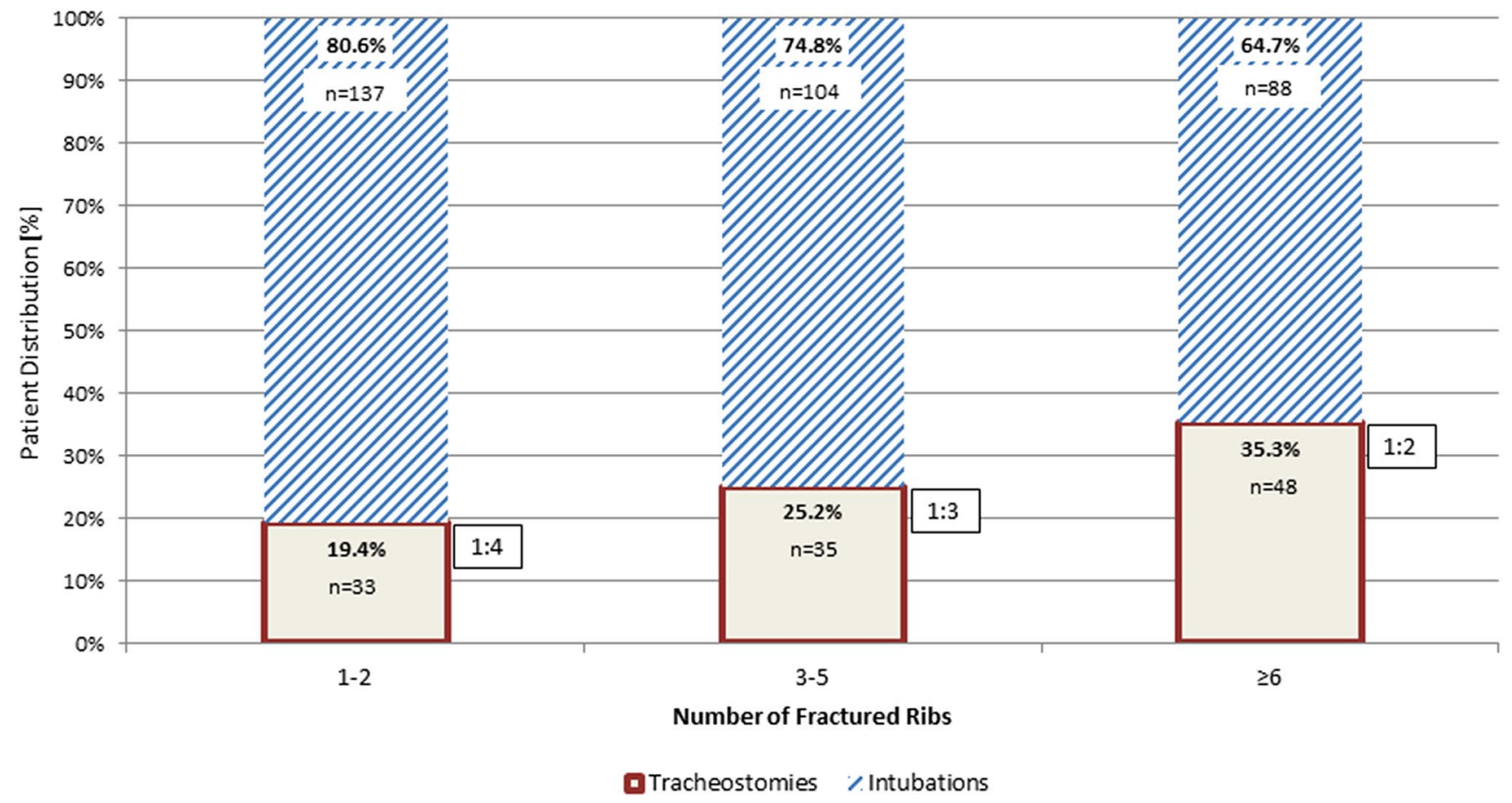

Fig. 2 Distribution of intubated and tracheostomized patients stratified by number of fractured ribs 
Table 2 Characteristics of tracheostomized patients in thoracic and extra-thoracic groups

\begin{tabular}{|c|c|c|c|}
\hline & Thoracic $(n=64)$ & $\begin{array}{l}\text { Extra-thoracic } \\
(n=60)\end{array}$ & $p$ values \\
\hline Age (yrs) & 52.9 & 47.6 & 0.1 \\
\hline Male gender $(\%)$ & $45.3 \%$ & $45.0 \%$ & 0.9 \\
\hline Injury severity score (ISS) & 26.0 & 29.2 & 0.05 \\
\hline Number of ribs fractured (Avg.) & 6.5 & 4.1 & $<0.001 *$ \\
\hline Number of total rib fractures (Avg.) & 7.6 & 4.3 & $<0.001^{*}$ \\
\hline Patients with displaced rib fractures & $37.5 \%$ & $23.3 \%$ & 0.09 \\
\hline Bilateral rib fractures & $34.9 \%$ & $23.7 \%$ & 0.2 \\
\hline Flail chest & $10.9 \%$ & $5.0 \%$ & 0.2 \\
\hline 1st rib fracture & $29.0 \%$ & $27.3 \%$ & 0.8 \\
\hline Glasgow coma scale (ED) & 11.5 & 7.0 & $<0.001^{*}$ \\
\hline TBI (intracranial hemorrhage) co-injuries & $21.9 \%$ & $71.7 \%$ & $<0.001^{*}$ \\
\hline Maxillofacial co-injuries & $12.5 \%$ & $53.3 \%$ & $<0.001 *$ \\
\hline Spine co-injuries & $53.1 \%$ & $60.0 \%$ & 0.4 \\
\hline Chest co-injuries (includes clavicle, sternal, scapula, diaphragm FXs) & $56.3 \%$ & $31.7 \%$ & $0.006 *$ \\
\hline Clavicle fracture & $29.7 \%$ & $23.3 \%$ & 0.4 \\
\hline Sternal fracture & $15.6 \%$ & $3.3 \%$ & $0.02 *$ \\
\hline Pulmonary co-injuries (includes hemo-/pneumothorax, pulmonary contusion) & $92.2 \%$ & $78.3 \%$ & $0.03 *$ \\
\hline Pulmonary contusion & $68.8 \%$ & $45.0 \%$ & $0.008^{*}$ \\
\hline Pneumonia & $42.2 \%$ & $41.7 \%$ & 1.0 \\
\hline Cardiovascular co-injuries & $15.6 \%$ & $16.7 \%$ & 0.9 \\
\hline Abdominal co-injuries & $45.3 \%$ & $28.3 \%$ & 0.05 \\
\hline Orthopedic co-injuries & $75.0 \%$ & $56.7 \%$ & $0.03 *$ \\
\hline Timing of tracheostomy (from intubation to tracheostomy) & 11.6 & 8.0 & $<0.001 *$ \\
\hline DMV (days) (from intubation to extubation) & 27.7 & 27.1 & 0.2 \\
\hline ICULOS (days) & 23.2 & 20.7 & 0.07 \\
\hline HLOS (days) & 41.3 & 39.3 & 0.5 \\
\hline
\end{tabular}

$F X$ fractures, TBI traumatic brain injury, DMV duration of mechanical ventilation, ICULOS intensive care unit length of stay, $H L O S$ hospital length of stay

*Denotes a significant difference

The optimal cut point based on ROC analysis for prediction of tracheostomy in the intubated thoracic and tracheostomized thoracic groups showed to be 6.5 days for the duration of intubation $(c=0.815$, sensitivity $=0.881$, specificity $=0.693$ ), and 4.5 for the number of fractured ribs $(c=0.721$, sensitivity $=0.644$, specificity $=0.668)$.

The multivariable logistic regression model to predict tracheostomy using ISS, pulmonary co-injuries, number of fractured ribs, duration of intubation and orthopedic co-injuries correctly classified $78.6 \%$ of cases. Prolonged intubation $(p<0.001$, adjusted odds ratio [OR]:1.18, 95\% confidence interval [CI]: 1.07-1.30) and increasing number of fractured ribs ( $p=0.001$, OR: $1.10,95 \%$ CI: $1.05-1.19)$ were associated with increased probability of tracheostomy in patients intubated for thoracic reasons.

The analysis of all tracheostomized patients stratified by the number of fractured ribs comparing thoracic and extrathoracic groups is presented in Fig. 3. As the number of fractured ribs increased, therefore amplifying the thoracic component of the trauma, there was a stepwise, two times increase in tracheostomies due to thoracic reasons from 1:2.2 (1-2 ribs fractured) to $1: 1$ (3-5 ribs fractured) to $2: 1$ ( $\geq 6$ ribs fractured). The difference was statistically significant between patients tracheostomized for thoracic reasons with 1-2 fractured ribs and patients with six or more ribs fractured.

Plotting ISS against the number of fractured ribs in all mechanically ventilated patients shows statistically significant increase in ISS values as the number of fractured ribs rises (Fig. 4).

Values for all chest trauma scoring systems in all groups are presented in Table 4 . All values were statistically significantly higher in patients with tracheostomy compared to intubated patients. Score values were even higher in the thoracic group of tracheostomized patients, reflecting the severity of thoracic trauma in this group. 
Table 3 Characteristics of patients intubated and tracheostomized for thoracic trauma

\begin{tabular}{|c|c|c|c|}
\hline & $\begin{array}{l}\text { Thoracic intubation } \\
(n=214)\end{array}$ & $\begin{array}{l}\text { Thoracic tracheostomy } \\
(n=64)\end{array}$ & $p$ values \\
\hline Age (yrs) & 51.9 & 52.9 & 0.7 \\
\hline Male gender $(\%)$ & $36.0 \%$ & $45.3 \%$ & 0.2 \\
\hline Injury severity score (ISS) & 20.2 & 26.0 & $<0.001^{*}$ \\
\hline Number of ribs fractured (Avg.) & 3.9 & 6.5 & $<0.001 *$ \\
\hline Number of total rib fractures (Avg.) & 4.5 & 7.6 & $<0.001^{*}$ \\
\hline Patients with displaced rib fractures & $23.4 \%$ & $37.5 \%$ & $0.03 *$ \\
\hline Bilateral rib fractures & $20.4 \%$ & $34.9 \%$ & $0.02 *$ \\
\hline Flail chest & $4.7 \%$ & $10.9 \%$ & 0.07 \\
\hline 1 st rib fracture & $18.7 \%$ & $29.0 \%$ & 0.08 \\
\hline Glasgow coma scale (ED) & 11.5 & 11.5 & 1.0 \\
\hline TBI (intracranial hemorrhage) co-injuries & $25.7 \%$ & $21.9 \%$ & 0.5 \\
\hline Maxillofacial co-injuries & $8.4 \%$ & $12.5 \%$ & 0.3 \\
\hline Spine co-injuries & $38.3 \%$ & $53.1 \%$ & $0.04 *$ \\
\hline Chest co-injuries (includes clavicle, sternal, scapula, diaphragm FXs) & $25.2 \%$ & $56.3 \%$ & $<0.001 *$ \\
\hline Clavicle fracture & $9.8 \%$ & $29.7 \%$ & $<0.001^{*}$ \\
\hline Sternal fracture & $4.7 \%$ & $15.6 \%$ & $0.003 *$ \\
\hline Pulmonary co-injuries (includes hemo-/pneumothorax, pulmonary contusion) & $78.0 \%$ & $92.2 \%$ & $0.01 *$ \\
\hline Pulmonary contusion & $46.7 \%$ & $68.8 \%$ & $0.002 *$ \\
\hline Pneumonia & $16.4 \%$ & $42.2 \%$ & $<0.001^{*}$ \\
\hline Cardiovascular co-injuries & $15.9 \%$ & $15.6 \%$ & 1.0 \\
\hline Abdominal co-injuries & $35.5 \%$ & $45.3 \%$ & 0.2 \\
\hline Orthopedic co-injuries & $54.7 \%$ & $75.0 \%$ & $0.004 *$ \\
\hline Timing of tracheostomy (from intubation to tracheostomy) & N/A & 11.6 & N/A \\
\hline DMV (days) (from intubation to extubation) & 6.0 & 27.7 & $<0.001^{*}$ \\
\hline ICULOS (Days) & 8.6 & 23.2 & $<0.001 *$ \\
\hline HLOS (Days) & 16.2 & 41.3 & $<0.001^{*}$ \\
\hline
\end{tabular}

$F X$ fractures, TBI traumatic brain injury, DMV duration of mechanical ventilation, ICULOS intensive care unit length of stay, $H L O S$ hospital length of stay

*Denotes a significant difference

Prediction values for tracheostomy by all chest trauma scoring systems are presented in Table 5.

\section{Discussion}

Overall, tracheostomized patients were indeed severely injured, had multiple rib fractures (5.2 fractured ribs on average), one-third had bilateral and displaced rib fractures, and $85 \%$ had pulmonary co-injuries. Our findings are in line with a recent report that polytrauma patients with rib fractures and pulmonary contusion are significantly more likely to be placed on a ventilator [17]. The results of our study demonstrate that overall a quarter of mechanically ventilated patients with rib fractures required tracheostomy (26.3\%). Our findings also show that an increased amount of fractured ribs is associated with an increase in tracheostomies versus intubations, as well as an increase in tracheostomies performed for thoracic reasons.

It was previously shown that up to $34 \%$ of ICU patients (medical, neurologic, trauma and general surgery ICUs) receiving mechanical ventilation for $>48 \mathrm{~h}$ undergo tracheostomy [18]. It was also reported that $21 \%$ of trauma ICU patients require tracheostomies [9]. Among our trauma patients with rib fractures who were mechanically ventilated for $>48 \mathrm{~h}, 32.5 \%$ underwent tracheostomy (124 of 381 patients).

Overall, the incidence of tracheostomy in patients with rib fractures in our study was $4.4 \%$. Reported incidence of tracheostomy in patients with rib fractures ranges widely from $0.3 \%$ to $79 \%[10,11,19]$. This broad span can be attributed to different cohorts of patients included into the studies and ever evolving treatment modalities. Althausen et al. presented $13.6 \%$ tracheostomy prevalence among flail chest patients with surgically stabilized rib fractures and 


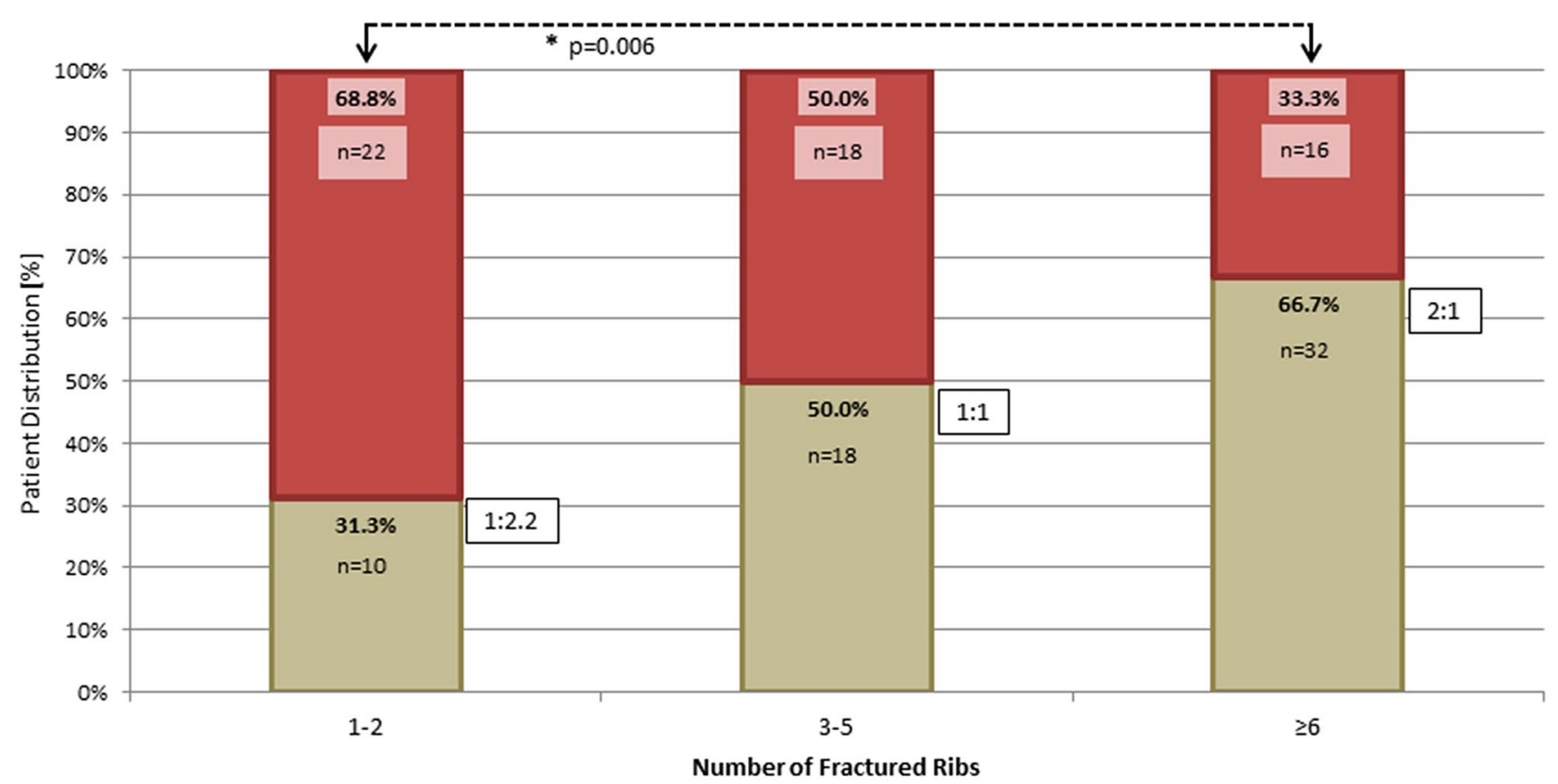

DThoracic Group Extrathoracic Group

Fig. 3 Proportion of thoracic versus extra-thoracic patients among all tracheostomized patients stratified by the number of fractured ribs

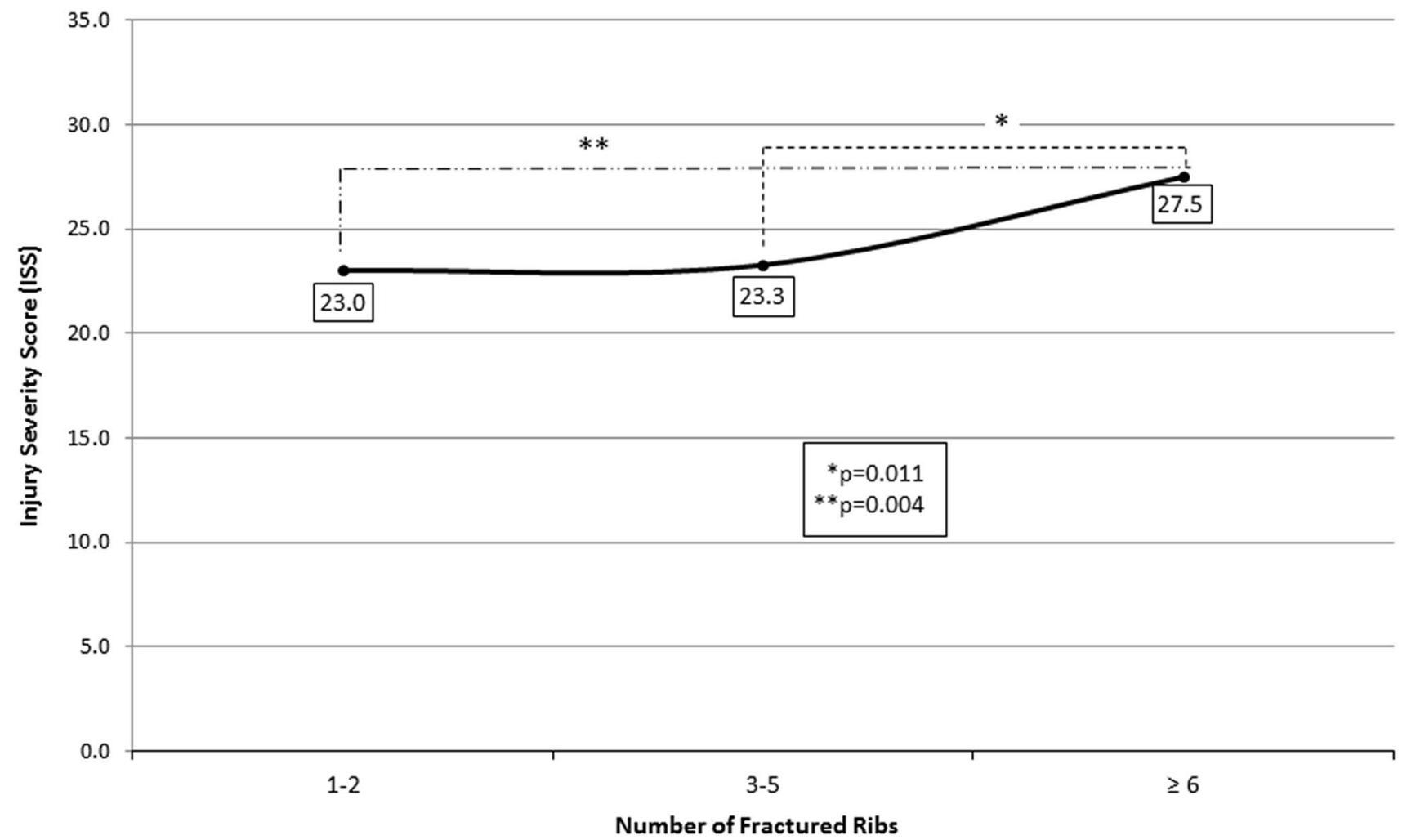

Fig. 4 Mean ISS versus number of fractured ribs in mechanically ventilated patients 
Table 4 Mean values of four rib fracture scoring systems in intubated and tracheostomized trauma patients

\begin{tabular}{|c|c|c|c|c|c|c|}
\hline & \multicolumn{3}{|c|}{ Mechanically ventilated patients } & \multicolumn{3}{|c|}{ Tracheostomized patients } \\
\hline & $\begin{array}{l}\text { Intubation } \\
(n=347)\end{array}$ & $\begin{array}{l}\text { Trache- } \\
\text { ostomy } \\
(n=124)\end{array}$ & $p$ values & $\begin{array}{l}\text { Thoracic } \\
(n=64)\end{array}$ & $\begin{array}{l}\text { Extra- } \\
\text { thoracic } \\
(n=60)\end{array}$ & $p$ values \\
\hline Rib fracture score (RFS) & 6.3 & 8.3 & $0.03 *$ & 11.0 & 5.9 & $<0.001 *$ \\
\hline Chest wall trauma score (CWTS) & 4.2 & 4.8 & $0.003 *$ & 5.5 & 4.5 & $0.001^{*}$ \\
\hline Chest trauma score (CTS) & 4.7 & 5.3 & $0.003 *$ & 5.9 & 4.7 & $0.001 *$ \\
\hline RibScore (RS) & 0.9 & 1.2 & $0.01 *$ & 1.5 & 1.0 & $0.02 *$ \\
\hline
\end{tabular}

*Denotes a significant difference

Table 5 Receiver operating characteristic curve values for prediction of tracheostomy

\begin{tabular}{lll}
\hline & $\begin{array}{l}\text { All tracheostomized }(n=124) \text { versus all intubated } \\
\text { patients }(n=347)\end{array}$ & $\begin{array}{l}\text { Tracheostomized thoracic only }(n=64) \\
\text { versus all intubated patients }(n=347)\end{array}$ \\
\hline Rib fracture score (RFS) & 0.582 & 0.685 \\
Chest wall trauma score (CWTS) & 0.622 & 0.702 \\
Chest trauma score (CTS) & 0.582 & 0.669 \\
RibScore (RS) & 0.575 & 0.630 \\
\hline
\end{tabular}

$39.3 \%$ in a non-operative cohort; however, authors excluded patients with GCS $<8$, severe spinal cord injuries, etc., and defined flail chest as fractures of 4 or more ribs at more than 2 sites [20]. Dehghan et al. reported an overall tracheostomy rate of $20.6 \%$ in patients with flail chest (defined as 3 or more ribs fractured in 2 or more locations), rate of $34.2 \%$ in patients with severe head injury, which was present in $15 \%$ of patients and tracheostomy rate of only $18.2 \%$ in patients without head injury [21]. Dehghan et al. also reported that the rates of tracheostomy were $22.1 \%$ and $11.2 \%$ in surgically treated flail chest patients and non-operatively managed patients, respectively [11].

Treatment of severely injured patients with chest trauma requires aggressive therapy, including: (1) effective pain management, (2) regional anesthesia (e.g., epidural analgesia), (3) posture therapy (seated position, if possible), (4) non-invasive ventilation, (5) bronchoscopy when needed, and (6) rib plating when indicated. Implementation of epidural analgesia was low in our institutions $(<2 \%)$. Many factors contributed to this: sedation and intubation before or within the first few hours of admission, severity of injury, inability to cooperate with positioning, restricted use of epidurals to 5 days due to infection risks, and contraindications on the use of anticoagulant venous thromboembolism prophylaxis in the presence of an epidural catheter.

SSRF among our patients was equally low (4\%) in tracheostomized and intubated groups. The indications for SSRF were: (1) flail chest ( $\geq 3$ consecutive rib fractures in two places), (2) multiple rib fractures $(\geq 5)$ especially with bicortical displacement, (3) fractured ribs acting as penetrating objects, (4) failure to control pain, (5) respiratory failure due to chest wall injury/pain, and (6) chest wall deformity and noticeable loss of thoracic volume. The main contraindications were severe traumatic brain injury and unstable cervical spine injury. These guidelines were based on the experience of participating trauma surgeons and also on the available literature $[6,22,23]$. Combination of multiple rib fractures (especially upper rib fractures) with ipsilateral clavicle fractures reduces the stability of the hemithorax. In the presence of concomitant ipsilateral rib fractures, there is a significant increase in midshaft clavicle fracture displacement rates, especially when upper ribs are involved [23-25]. Our tracheostomized patients in comparison with intubated patients had statistically significantly higher incidence of clavicle fractures, which also coalesced with a statistically higher number of fractured ribs.

Several chest trauma scores were described to predict tracheostomy administration. RibScore was developed to predict adverse pulmonary outcomes such as pneumonia, acute respiratory failure and the need for tracheostomy. It was reported that RibScore was significantly linearly correlated with tracheostomy and patients with tracheostomy had median RibScore statistically significantly higher than those who did not have a tracheostomy [15]. CWTS and CTS were developed to identify patients at a greater risk for respiratory failure, need for intubation, ICU admission, and mortality $[13,14]$. It was found that prevalence of tracheostomy was 2.5 times higher in patients whose score had reached the cut point value. RFS was developed to predict the need for respiratory support, mobilization, pain management, and the author developed a management protocol where increasing ranges of score were linked to the increasing rigor of 
recommended treatment [12]. In our study, all rib scoring systems' mean values were statistically significantly higher in patients with a tracheostomy (Table 4). These findings are also in line with tendencies described by the authors of scoring systems.

ROC AUC analysis for prediction of tracheostomy by all scoring systems in all mechanically ventilated patients yielded unconvincing results (Table 5), ranging from "failed" to "poor," which can be partly explained by the lack of variables in the scoring systems that are also directly related to the indications for tracheostomy (MFM/ TBI/CVT). The other possible reason for the discrepancy is that cohorts of patients from which scoring systems were developed were not adjusted for isolated thoracic trauma/rib fractures. For example, RFS was developed from a cohort that included only $6 \%$ of patients with isolated rib fractures and $8 \%$ with a TBI, while RS cohort had $47 \%$ with isolated rib fractures, $27.8 \%$ with a head injury and $18.7 \%$ with facial fractures $[12,15]$. When we applied scoring systems to predict tracheostomy in the thoracic groups of patients, their predictive values increased from "poor" to "fair," as compared to those in the extra-thoracic groups (Table 5). There are several other scoring systems available such as Stroke-Related Early Tracheostomy Score and Acute Physiologic and Chronic Health Evaluation score; however, they were not designed to address patients with thoracic trauma and multiple rib fractures in particular [26, 27].

Comparison between intubated and tracheostomized patients, between patients tracheostomized for thoracic reasons versus extra-thoracic reasons and between patients intubated for thoracic reasons versus patients tracheostomized for thoracic reasons (Tables 1, 2, 3) revealed that the main distinguishing variables were the number of fractured ribs, duration of intubation, presence of pneumonia or pulmonary contusion and orthopedic co-injuries. These variables were further confirmed to play a role in the prediction of tracheostomy by the multivariable logistic regression analysis. Orthopedic co-injuries can be speculated to result in reduced mobility, consequently prolonging patient's immobilization (e.g., femoral fractures, pelvic fractures) with all known negative consequences. Therefore, we suggest that thoracic trauma patients with $\geq 5$ ribs fractured, intubated for a week and with pulmonary contusion or pneumonia, should be considered candidates for tracheostomy, especially if they also have orthopedic co-injuries. We think that these observations could be included into the clinical decision making.

\section{Limitations}

Our study has several limitations. This is a retrospective chart review study with known shortcomings of analysis of the prerecorded data [28]. Patients' cohort was limited to two level 1 trauma centers with inherently more severely injured patients. The number of patients who underwent tracheostomy was limited and was lower than those who had an endotracheal intubation only. Since the rates of epidural analgesia administration and surgical stabilization of rib fractures were $1.6 \%$ and $4.0 \%$, respectively, we did not control for the effects of these procedures on the outcomes. The degree of pulmonary contusion was not quantified, as this data was not available from the review charts.

\section{Conclusions}

A quarter of mechanically ventilated trauma patients with rib fractures required tracheostomy. Tracheostomized compared to intubated patients were more severely injured with more ribs fractured and were intubated longer. Half of the tracheostomized patients with rib fractures underwent tracheostomy due to severe brain injury, maxillofacial-mandibular or cervical vertebrae trauma. An increased number of fractured ribs were associated with an increase in tracheostomies and thoracic indications for tracheostomy.

\section{Compliance with ethical standards}

Conflict of interest Author Alexander Fokin, Author Joanna Wycech, Author Kyle Chin Shue, Author Ryan Stalder, Author Jose Lozada, and Author Ivan Puente declare that they have no conflict of interest.

Ethical statement This manuscript has not been published previously or submitted elsewhere for publication and will not be sent to another journal until a decision is made concerning publication by the European Journal of Trauma and Emergency Surgery.

Ethical approval This retrospective cohort study was approved by IRB. All procedures performed in the study involving human participants were in compliance with the ethical standards of the institutional and/ or national research committee and with the 1964 Helsinki Declaration and its later amendments or comparable ethical standards.

Informed consent The waiver of informed consent was granted by IRB.

\section{References}

1. Sirmali M, Turut H, Topcu S, et al. A comprehensive analysis of traumatic rib fractures: morbidity, mortality and management. Eur J Cardiothorac Surg. 2003;24(1):133-8.

2. Ziegler DW, Agarwal NN. The morbidity and mortality of rib fractures. J Trauma. 1994;37(6):975-9.

3. Jones KM, Reed RL 2nd, Luchette FA. The ribs or not ribs: Which influences the mortality? Am J Surg. 2011;202:598-604.

4. Flagel BT, Luchette FA, Reed RL, et al. Half-a-dozen ribs: the breakpoint for mortality. Surgery. 2005;138(4):717-23.

5. Emond M, Sirois MJ, Guimont C, et al. Functional impact of a minor thoracic injury. Ann Surg. 2015;262(6):1115-22. 
6. Shulzhenko NO, Zens TJ, Beems MV, et al. Number of rib fractures thresholds independently predict worse outcomes in older patients with blunt trauma. Surgery. 2017;161(4):1083-9.

7. Battle CE, Hutchings $\mathrm{H}$, Evans PA. Risk factors that predict mortality in patients with blunt chest wall trauma: a systematic review and meta-analysis. Injury. 2012;43(1):8-17.

8. Flores-Funes D, Lluna-Llorens AD, Jimenez-Ballester MA, et al. Is the number of rib fractures a risk factor for delayed complications? Eur J Trauma Emerg Surg. 2018. https://doi.org/10.1007/ s00068-018-1012-x.

9. Arabi Y, Haddad S, Shirawi N, Shimemeri AA. Early tracheostomy in intensive care trauma patients improves resource utilization: a cohort study and literature review. Crit Care. 2004;8(5):R347-52.

10. Kane ED, Jeremitsky E, Bittner KR, Kartiko S, Doben AR. Surgical stabilization of rib fractures: a single institution experience. $J$ Am Coll Surg. 2018;226(6):961-6.

11. Dehghan N, Mah JM, Schemitsch EH, Nauth A, Vicente M, McKee MD. Operative stabilization of flail chest injuries reduces mortality to that of stable chest wall injuries. J Orthop Trauma. 2018;32(1):15-21.

12. Easter A. Management of patients with multiple rib fractures. Am J Crit Care. 2001;10(5):320-7.

13. Pressley CM, Fry WR, Philp AS, Berry SD, Smith RS. Predicting outcome of patients with chest wall injury. Am J Surg. 2012;204(6):910-3.

14. Chen J, Jeremitsky E, Philp F, Fry W, Smith RS. A chest trauma scoring system to predict outcomes. Surgery. 2014;156(4):988-94.

15. Chapman BC, Herbert B, Rodil M, et al. RibScore: a novel radiographic score based on fracture pattern that predicts pneumonia, respiratory failure, and tracheostomy. J Trauma Acute Care Surg. 2016;80(1):95-101.

16. Fokin AA, Wycech J, Crawford M, Puente I. Quantification of rib fractures by different scoring systems. J Surg Res. 2018;229(9):1-8.

17. Miller C, Stolarski A, Ata A, et al. Impact of blunt pulmonary contusion in polytrauma patients with rib fractures. Am J Surg. 2019. https://doi.org/10.1016/j.amjsurg.2019.01.027.
18. Quality of life after mechanized ventilation in the elderly study investigators. 2-month mortality and functional status of critically ill adult patients receiving prolonged mechanical ventilation. Chest. 2002;121(2):549-58.

19. Beks RB, Peek J, de Jong MB, et al. Fixation of flail chest or multiple rib fractures: current evidence and how to proceed. A systematic review and meta-analysis. Eur J Trauma Emerg Surg;. 2018. https://doi.org/10.1007/s00068-018-1020-x.

20. Althausen PL, Shannon S, Watts C, et al. Early surgical stabilization of flail chest with locked plate fixation. J Orthop Trauma. 2011;25(11):641-7.

21. Dehghan N, de Mestral C, McKee MD, Schemitsch EH, Nathens A. Flail chest injuries: a review of outcomes and treatment practices from National Trauma Data Bank. J Trauma Acute Care Surg. 2014;76(2):462-8.

22. Pieracci FM, Majercik S, Ali-Osman F, et al. Consensus statement: surgical stabilization of rib fractures rib fracture colloquium clinical practice guidelines. Injury. 2017;8(2):307-21.

23. Fokin AA, Wycech J, Weisz R, Puente I. Outcome analysis of surgical stabilization of rib fractures in trauma patients. J Orthop Trauma. 2019;33(1):3-8.

24. Stahl D, Ellington M, Brennan K, Brennan M. Association of ipsilateral rib fractures with displacement of midshaft clavicle fractures. J Orthop Trauma. 2017;31(4):225-8.

25. Fokin A, Wycech J, Picard E, Weisz R, Puente I. Is first rib fracture a culprit or a sign of injury severity? It is both. J Orthop Trauma. 2018;32(8):391-6.

26. Casamento A, Bailey M, Robbins R, et al. Patient characteristics, incidence, technique, outcomes and early prediction of tracheostomy in the state of Victoria, Australia. J Crit Care. 2018;44:278-84.

27. Alsherbini K, Goyal N, Metter EJ, et al. Predictors for tracheostomy with external validation of the stroke-related early tracheostomy score (SETscore). Neurocrit Care. 2019;30(1):185-92.

28. Kaji AH, Schringer D, Green S. Looking through the retrospectoscope: reducing bias in emergency medicine chart review studies. Ann Emerg Med. 2014;64(3):292-8. 